Forum 2018 · 33:152

https://doi.org/10.1007/s12312-018-0422-3

(c) Springer Medizin Verlag GmbH, ein Teil von Springer Nature 2018

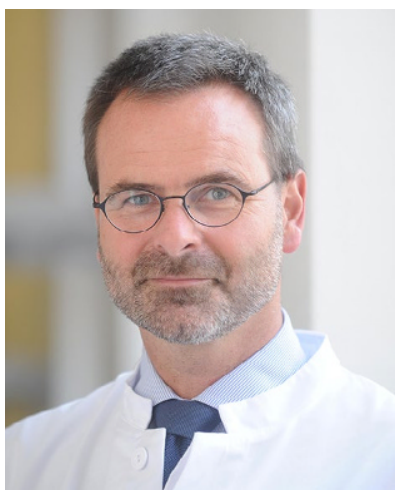

Die Politik hat einen Neustart in der Pflege angekündigt. Der Blick auf die im Koalitionsvertrag vereinbarten Maßnahmen ist aber eher ernüchternd. Rund 8000 zusätzliche neue Pflegestellen will die Bundesregierung schaffen - ein Tropfen auf den heißen Stein angesichts der rund 100.000 fehlenden Pflegekräfte allein in deutschen Krankenhäusern. Doch schon die Besetzung dieser 8000 Stellen dürfte angesichts des Mangels an qualifizierten Pflegekräften nicht einfach sein.

Der Pflegeberuf hat in den letzten Jahren zunehmend an Attraktivität eingebüßt. Das bestätigt auch der CARE Klima-Index 2017 vom Psyma-Marktforschungsinstitut und dem Deutschen Pflegerat. Die Gründe: zu wenig Zeit für eine bedarfsorientierte Pflege und mangelnde Wertschätzung. $56 \%$ der professionell Pflegenden beurteilten den gesellschaftlichen Stellenwert ihrer Profession niedriger als den anderer Berufsgruppen. 80\% fanden die personelle Ausstattung ihrer Einrichtung nicht ausreichend. Darunter leidet vor allem die Qualität der pflegerischen Versorgung.

Wie wirkt sich dieser Trend auf die Pflege onkologischer Patienten auf? Die Behandlungsstrategien in der Onkologie

\title{
Was muss sich ändern in der onkologischen Pflege?
}

werden zunehmend komplexer und erfordern mehr Fachlichkeit, auch von der Pflege. Gefragt sind daher - über die Grundpflege hinaus - vor allem Fachkenntnisse im Umgang mit Nebenwirkungen und mit unterstützenden Maßnahmen, dazu die Fähigkeit zum sensiblen Umgang mit den Patienten sowie Beratungskompetenz. Onkologische Fachpflegekräfte sind für diese Aufgaben durch eine zweijährige Zusatzausbildung qualifiziert. Aufgrund des Pflegenotstands und aus Gründen der Wirtschaftlichkeit werden sie aber oft nicht für die Aufgaben eingesetzt, für die sie eigentlich ausgebildet sind, betonte Kerstin Paradies, Vorstandssprecherin der Konferenz Onkologischer Kranken- und Kinderkrankenpflege auf dem DKK 2018. Die große Teilnehmerzahl in den Pflegesitzungen auf dem DKK machte den Diskussionsbedarf deutlich.

Was muss sich ändern? Pflegerische Leistungen brauchen eine ausreichende Gegenfinanzierung und ohne Personalbesetzungsstandards sind Verbesserungen kaum möglich. Den Vorstoß der letzten Bundesregierung, verbindliche Pflegepersonaluntergrenzen für pflegesensitive Bereichen einführen zu wollen, finde ich allerdings problematisch. Pflegeschlüssel sollten sich am tatsächlichen Bedarf der Patienten orientieren, nicht an ausgewählten Versorgungsbereichen.

Wer Menschen für den Pflegeberuf begeistern möchte, muss auch die Frage nach der Attraktivität des herkömmlichen Berufsbilds stellen. In vielen anderen Gesundheitssystemen hat die Pflege einen höheren Stellenwert, weil dort Pflegekräfte, nach entsprechender Qualifikation, mit weiterreichenden Kompetenzen ausgestattet sind als hierzulande. Auch in Deutschland wollen viele Pflegende gerne mehr Verantwortung übernehmen, werden vom System aber ausgebremst. Die öffentliche Debatte verkürzt das Problem auf das Gehalt. Das eigentliche Problem liegt aber in der mangelnden Wertschätzung und den nicht vorhandenen Aufstiegschancen für Qualifizierte. Der Akademisierungsgrad der Pflege von weniger als einem Prozent ist im Vergleich $\mathrm{zu}$ anderen Ländern wesentlich zu niedrig; darüber hinaus wandern akademisch qualifizierte Pflegekräfte in die Administration ab. Stattdessen brauchen wir studierte Pflegekräfte am Patienten, vor allem in der Onkologie, wo es auf eine qualitativ hochwertige Pflege ankommt. Aber auch in vielen anderen Bereichen (etwa in der Vorsorge, Schwangerenbetreuung, Tumornachsorge) können qualifizierte Pflegekräfte zum Beispiel mit einem Bachelorabschluss ärztliche Tätigkeiten übernehmen. Ein derartig verändertes Berufsbild ist deutlich attraktiver und man verhindert so, dass über Pflege nur im Zusammenhang mit „mehr Geld“ geredet wird.

Pflegeschlüssel und die Kompetenz der Pflegenden wirken sich nachhaltig auf die Qualität der Krebsversorgung aus Als interdisziplinäre und interprofessionelle Fachgesellschaft müssen wir uns ernsthaft und kurzfristig damit auseinandersetzen. Die Beiträge in diesem Heft sind ein guter Einstieg.

Ihr

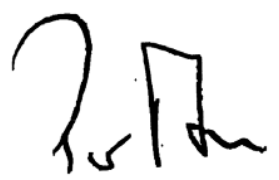

Peter Albers

Präsident der Deutschen Krebsgesellschaft 